\title{
Detection of slag inclusions using infrared thermal imagining system
}

\author{
Wojciech Jamrozik $^{1 *}$, Jacek Górka \\ ${ }^{1}$ Silesian University of Technology, Faculty of Mechanical Engineering, Department of Fundamentals \\ of Machinery Design, 18A Konarskiego Street, 44-100, Gliwice, Poland \\ ${ }^{2}$ Silesian University of Technology, Faculty of Mechanical Engineering, Department of Welding \\ Engineering, 18A Konarskiego Street, 44-100, Gliwice, Poland
}

\begin{abstract}
Assuring high quality of welded joins is a vital task in many industrial branches also when joints are made manually. It is the case metal-arc welding with covered electrode. One of main imperfection, that can occur in this process is slag inclusion. In the paper an method for detection of slag inclusion in multipass manual welding is proposed and validated. The key idea of the method is that small temperature disturbances will be noticeable in consecutive cross-section of joint in the cooling pass. Temperature distribution weld face was measured with longwave infrared camera (LWIR). For consecutive cross-section made in IR representation of joint differences in mean temperature was calculated to assess the cooling rate directly after the elements were welded. It can be made because on each thermogram the whole joint is visible, thus position of electrode in time can be easily marked. Results of slag inclusion detection were compared with radiographic images of made joints. In the future additional studies will be performed in order to generalize proposed method to wider group of materials and for more complex welds.
\end{abstract}

\section{Introduction}

Manual welding with coated electrode (MMAW - Manual Metal Arc Welding - 111) is a process of permanent joining of metals, consisting in melting an additional material, called a binder, and the edges of the base material using a heat source [1]. The method of welding with coated electrodes is used to connect generally all steel grades (unalloyed steels, highstrength steels, high-alloy steels), copper, nickel and even grey cast iron for repair welding [2]. The welding process can also be carried out under water $[3,4]$. In recent years, coated electrodes have also appeared on the market, the binder of which has a nanocrystalline structure, with very high abrasion resistance, which is why they are used to connect wearresistant plates [5]. The source of heat in this method is the electric arc glowing between the coated electrode core and the surface of the welded material. The temperature of this arc is between $2400^{\circ} \mathrm{C}$ and $5000^{\circ} \mathrm{C}$. The arc is struck by the welder by shorting the electrode to the material and then quickly withdrawing it to the appropriate height. The arc length is equal to this height and should be equal to the diameter of the electrode core. After the arc

* Corresponding author: wojciech.jamrozik@polsl.pl 
ignites, a weld pool is formed under the electrode, consisting of liquid metal derived from the base material and electrode core as well as liquid slag resulting from the melting of the coating. The melting cover, apart from slag, also produces a gas shield consisting of $\mathrm{CO}_{2}$, $\mathrm{CO}$ and $\mathrm{H}_{2} \mathrm{O}$. The role of the slag flowing out onto the surface of the liquid metal and the gas shield is to cut off the weld from the access of nitrogen and oxygen from the air, refining the liquid metal pool, introducing alloying elements, forming the weld face and slowing down the cooling rate $[6,7]$. The liquid weld pool solidifies forming a weld and a layer of slag on it, which must be mechanically removed before applying the next stitch. Incorrect removal of slag in the case of multi-stitch welding is often the cause of welding incompatibilities from the group of 300 called: slag inclusion. Slag remelting with electric arc welding does not eliminate this problem. In order to ensure the quality of the welded joint, it is necessary to properly clean the weld, often requiring the use of mechanical devices. In the case of large slag inclusions, this welding incompatibility becomes a disadvantage and the welded joint cannot be put into service, but requires repair, which significantly increases production costs. In industry, the following 4 major types of welding electrode coatings are the most commonly used. They are: Rutile Coating, Basic or low H2 Coating, Cellulosic Coating, Iron oxide Coating. Rutile electrodes are standard electrode for universal use, with fine to medium droplet transfer and good mechanical properties of joints. Basic electrodes can be used where high mechanical properties are required. They are characterized with average to coarse droplet transfer and, what is one of main disadvantages, poorly detaching slag (ewm-group.com). Slag inclusion possibility is determined by several factors. Not only electrode coating selection, but also welder skills and the quality of material preparation is important. In MMA welding, that is performed manually human factors plays key role. As many scientists and companies are working on methods and applications for on-line monitoring of welding process stability and joints quality, there are not many that took into consideration MMA welding [8]. A vast of approaches is devoted to the robotized or automated stands, where the number of process freedom degrees is limited. There are methods in which electric process signals, thermography, vision light cameras, spectrometers or acoustic emission sensors were applied [9]. As for MMA welding on-line quality checking is highly rare, different nondestructive testing (NDT) methods are used after the joining process is completed [10, 11]. Most popular are ultrasonic examinations as well as radiographic (X-ray) testing used mainly off-line, but also in some applications and environments in on-line constant monitoring manner $[12,13]$. Radiographic test results are highly reliable and easier to interpret as the ultrasonic examination results. For example low-density inclusions of materials like slag will look darker on the radiographic image than base metal or welded seam. In the paper a method that can be used on-line for slag inclusion detection in MMA welding is described. It is based on the analysis of IR images (thermograms) sequences [14, 15]. The features describing the cooling process in the welding pool is used. All analysis are made in the signal domain, without frequency domain transformations.

\section{Analysis of thermogram sequence}

The proposed method is based on an analysis of thermogram sequences taken during the MMA welding. The camera optical axe is perpendicular to the line of seam. Additionally the angle between camera axe and specimen surface should be greater than $60^{\circ}$, to limit the influence of angular emissivity on the results and also to assure good visibility and symmetry of weld face and welding pool (Fig. 1). Each thermogram was reduced to subimage that contains ROI (Region of Interest) in which the welding groove, joint and the heat affected zone are represented. In the ROI three types of geometrical zones (Fig. 2) were selected to calculate features that can be used to estimate welding process and seam in 
a spatial - temporal domain. Rectangles covering welding groove area $\left(\mathrm{A}_{\mathrm{wg}}\right)$ as well as rectangle that is narrower in comparison to the first one $\left(\mathrm{A}_{\mathrm{rwg}}\right)$ were superimposed on the thermogram ROI.

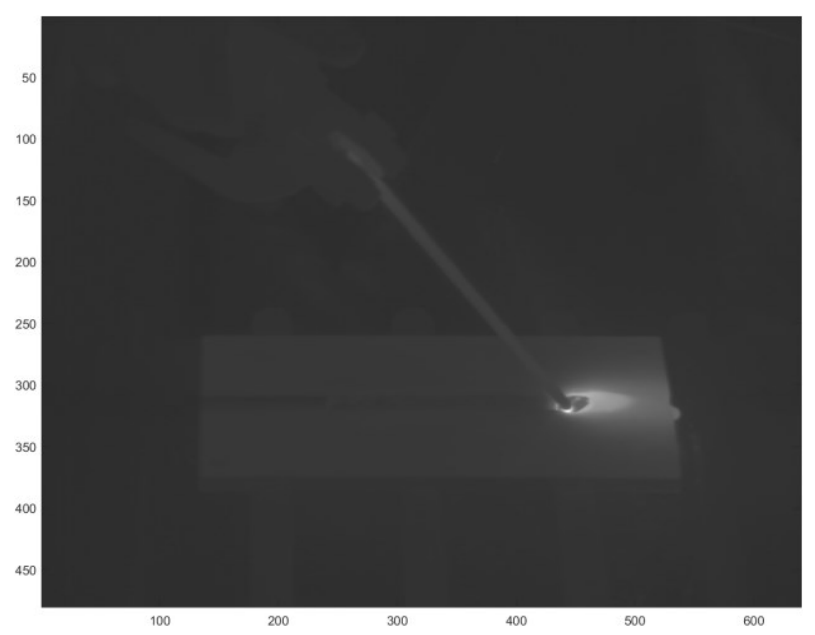

Fig. 1. Exemplary thermogram of MMA process.

Those rectangles were used to cut off outside part of the ROI, and only the part that remains has been processed and analysed. A mean horizontal profile, describing the temperature change in along the welding path was calculated for both $A_{w g}$ and $A_{r w g}$. The width of reduced rectangle was estimated as a result of entropy analysis of the temperature values in the rectangular region. A rectangle with the lowest decrease of entropy in comparison to the Awg was selected. The last geometrical object was a line crossing the point with maximal temperature in thermogram. In case of more points with the same value, the closest point to the bottom edge of welding groove was chosen. Along drawn line horizontal profile was taken.

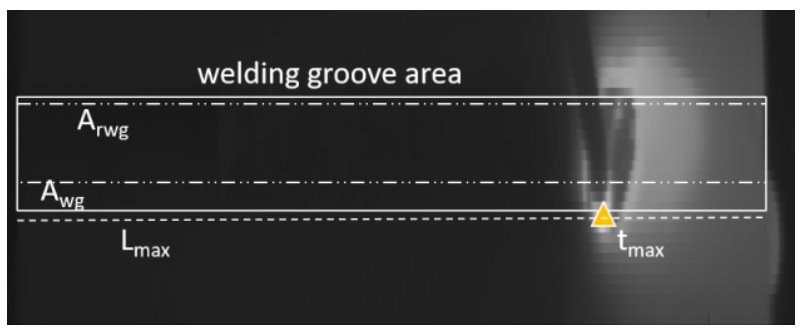

Fig. 2. ROIs marked on the exemplary IR image.

For all thermograms in sequence mean profiles were extracted. The set of profiles, gathered in a $2 \mathrm{D}$ array was considered as a complete representation of the realization of MMA welding. On a heatmap all mean profiles of one feature type extracted for one sequence of IR images several meaningful areas can be found (Fig. 3, Fig. 4). First area is the region of welding pool, that is in front of the electrode tip (Fig. 3a). Then the seemingly coolest area (Fig. 3b) is the region where the elected tip was observed. Next one is the welding pool area (Fig. 3c) and the last one is the cooling zone of solidified seam (Fig. 3d). It has to be noticed, that because of the different ability of surface to emit energy in the form of thermal radiation (different emissivity, for the molten steel $\varepsilon<0.1$ while for the solid steel $\varepsilon>0.5$ ) the molten metal area is apparently colder form the solidified seam part. 


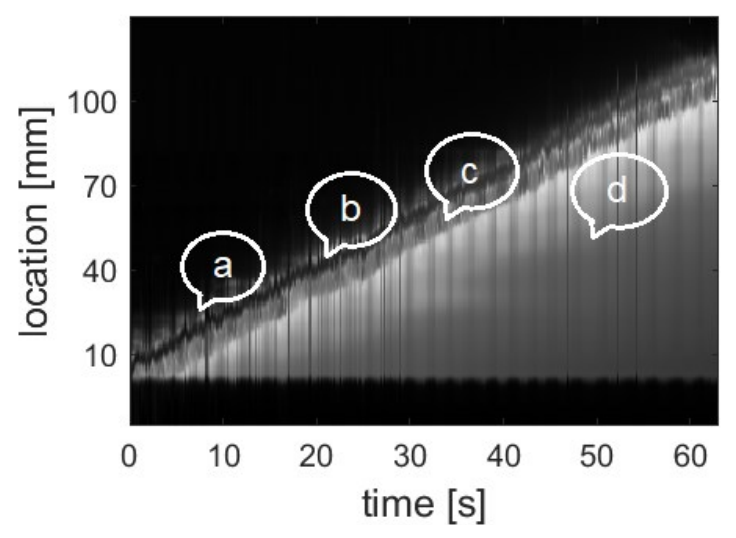

Fig. 3. Exemplary welding sequence heatmap extracted from $A_{\text {rwg }}$ ROIs with principal zones markers (described in text).

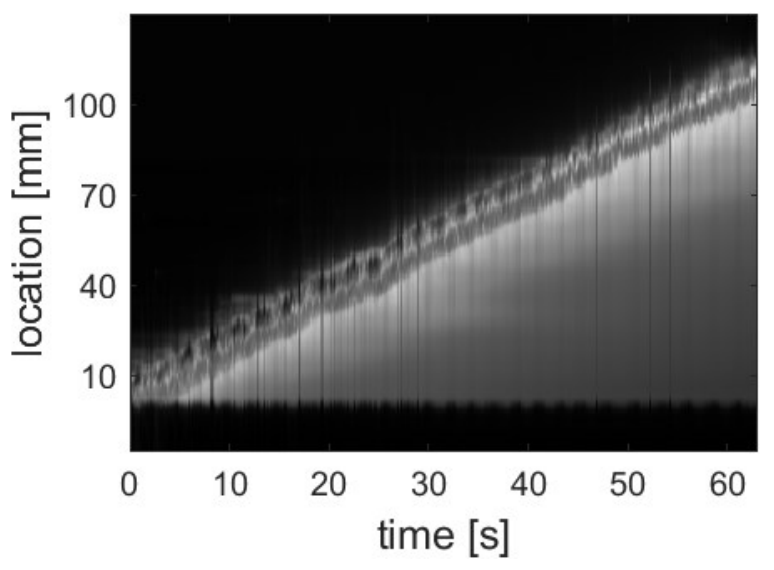

Fig. 4. Exemplary welding sequence heatmap extracted from $A_{w g}$ ROIs.

For the heatmap where profiles only for line crossing max pixel (Fig. 5) the small oblique movement of the electrode tip is clearly visible. The averaging of the temperature in $A_{w g}$ or $A_{\text {rwg }}$ area gave better results as it was in the case of $L_{\max }$ feature, especially in terms of process stability assessment. The electrode tip path is clearly visible in the area based features. To perform detection and localization of slag inclusion it was necessary to check variations of welding speed. To find correspondence between the features calculated for certain time of the welding process and location of electrode the welding speed has to be constant. To check it image processing procedure was proposed. The map composed of $\mathrm{A}_{\mathrm{rwg}}$ mean profiles was used. First processing stage was to cut off part of profile that is above the maximal temperature (in the welding pool area). 


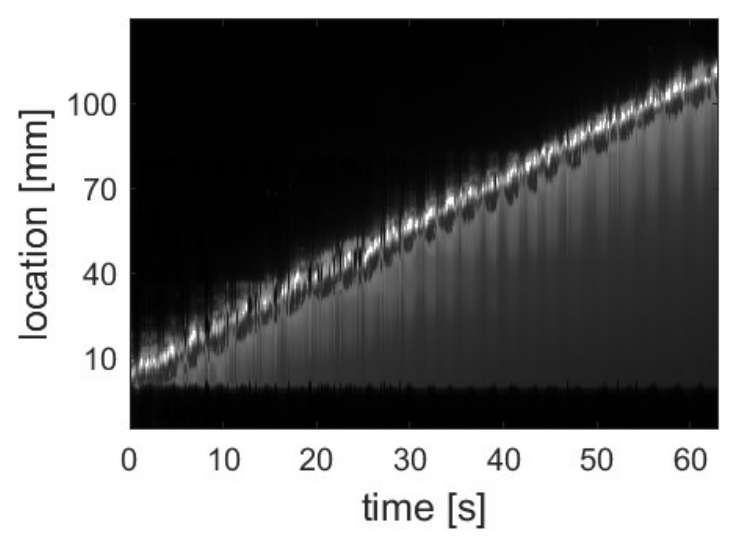

Fig. 5. Exemplary welding sequence heatmap extracted from $\mathrm{L}_{\max }$ profiles.

Obtained map (Fig. 6) is used to calculate location/time function plot. This is made by taking the envelope like signal from the remained $\mathrm{A}_{\mathrm{rwg}}$ profiles. Linearity check was made by fitting line to the plot using simple linear regression. $\mathrm{R}^{2}$ (a goodness-of-fit measure for linear regression models) parameter was used to evaluate the welding speed variations. If the $\mathrm{R}^{2}$ value is near to one it can be stated that line model is well fitted to data what can be interpreted that tested part was welded with constant speed.

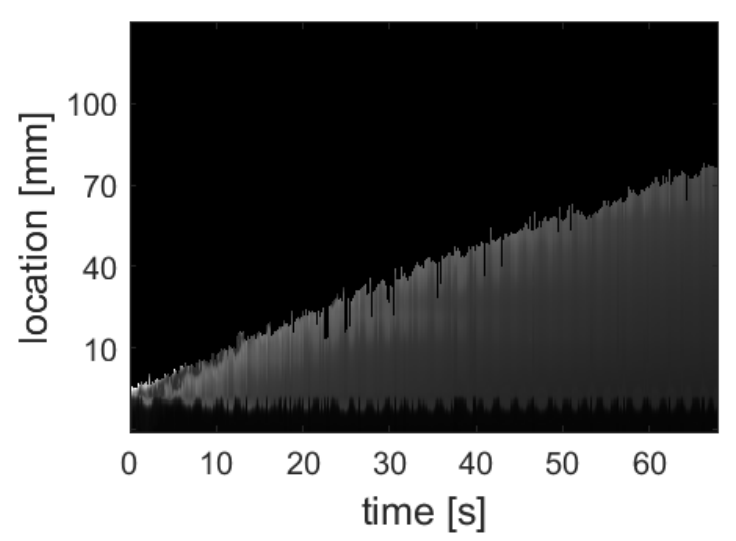

Fig. 6. Exemplary welding sequence heatmap extracted from $A_{r w g}$ ROIs prepared for welding speed stability assessment.

After checking the welding speed constancy detection signal $t_{c}$ was generated. Taking into consideration the regression speed line for the maximal values for each time moment, a part of profile extracted. The part that remains was started in the max temp. location towards the solidified metal zone. The new profile had fixed length. After preliminary studies it was set to 30 pixels, what is about $8 \mathrm{~mm}$ of the seam length (Fig. 7). In tan be seen that now, the electrode tip path is near to horizontal line. Next the values in inch vertical profile (for certain time) were averaged, to find mean value in each profile (tc signal was obtained). To remove some noise from the result, the tc signal was smoothed using the moving average (window width 4). Detection and localization of inclusion was made in a threshold range. The threshold was estimated based on the standard deviation analysis of signal estimated for all samples without intentionally introduced slag inclusion. The range was set as the $\pm 5 \%$ of the threshold value. 


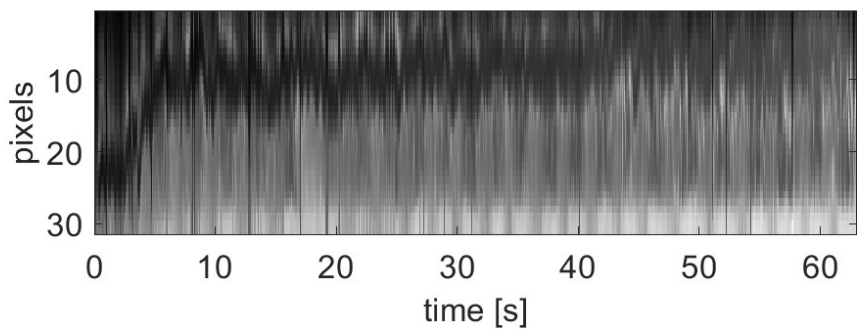

Fig. 7. Heatmap of reduced and linearized temperature profiles for whole welding realization.

\section{Case study}

In the performed experiment there were eight samples welded by a highly experienced welder. The MMA process was performed manually and the welder was trying to keep constant welding arc length and constant welding speed. To join each sample two seems were made. To introduce inconsistencies to the welded joint, especially to produce slag inclusions in one group of samples the slag that was created on the face of first seam was not removed. In the second group of samples slag layer was mechanically removed and the weld face was cleared. All samples were joined with the use of basic coated electrodes $E 42$ $4 \mathrm{~B} 42 \mathrm{H} 5$ with a diameter of $3.2 \mathrm{~mm}$. Before welding, the electrodes were dried at $300^{\circ} \mathrm{C}$ for two hours. It was necessary to obtain low moisture content in electrode what is demanded to reach high crack resistance in joint. Coating of basic electrodes involves big amount of alkaline-earth metals carbonates. Proper use of those electrodes leads to low content of oxides inclusions and provide relatively high plasticity of weld metal. Nevertheless electrodes with basic coating have also some deficiencies: sensitivity to porosity formation, sensitivity to contaminations, moreover the produced slag is hard to remove in the production process. To simulate real life production problems, this type of electrode was chosen. The welding current was 120A DC+. Each of test samples was of size $100 \times 120 \times 10 \mathrm{~mm}$. Measurement of temperature distribution in joints were performed using uncooled microbolometer IR camera VarioCam HR head 600, with spatial resolution of $640 \times 480 \mathrm{px}$ and acquisition frequency of $60 \mathrm{fps}$. For all observations constant emissivity was used. There were no emissivity variations as a function of temperature or observation angle considered. It was a deliberate assumption, because slag and other inclusions, gas pores and other contaminations will have different properties than welded metal bringing nonhemogenic areas into welding pool or solidified joint.

\section{Results}

Two samples were chosen to present differences between seam with slag inclusions and the correct one. Specimens were selected according to results of radiographic testing. Slag inclusions were revealed in X-ray testing, and the seam containing most of slag inclusions was chosen. Both samples were joined with seams that were $110 \mathrm{~mm}$. The radiographic examination revealed that there were also other welding inconsistencies, like incomplete root penetration (imperfection no. 4021) are present in both samples. Nevertheless those defects did not affect results of proposed procedure. 


\subsection{Sample with slag}

For the sample with slag inclusion two area with defects were found (Fig. 8b, marked in circles) at 4th and 7th $\mathrm{cm}$ of joint length. Slag is clearly visible in the form of darker, circular areas in the radiogram. In order to assess the welding speed linearity check procedures was applied on the heatmap (Fig. 9).

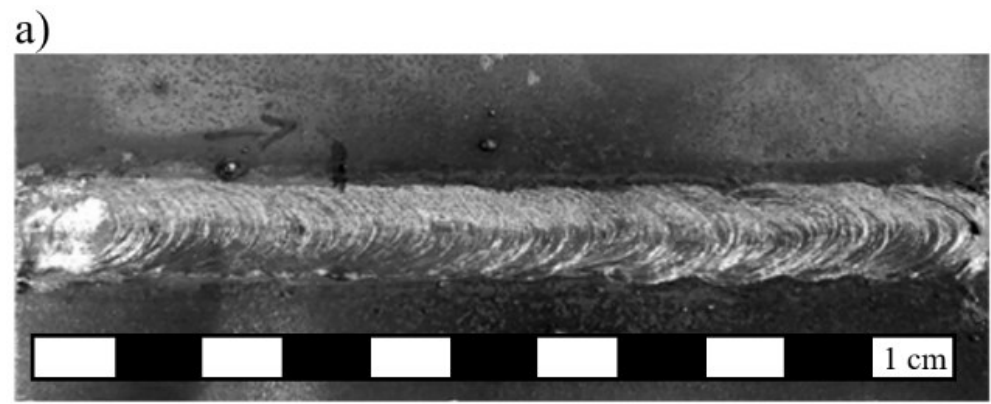

b)

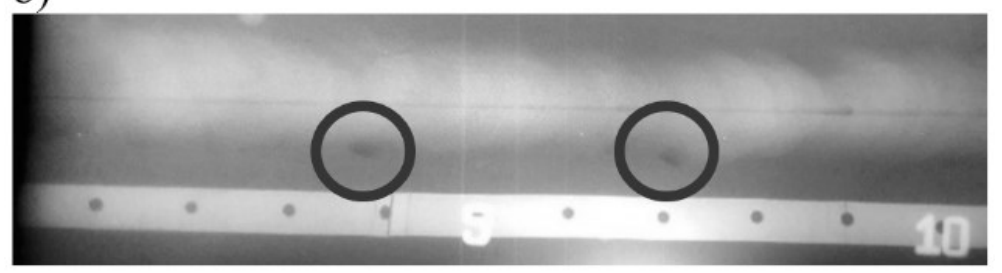

Fig. 8. Welded sample with slag inclusions: a) face view, b) radiogram of joint.

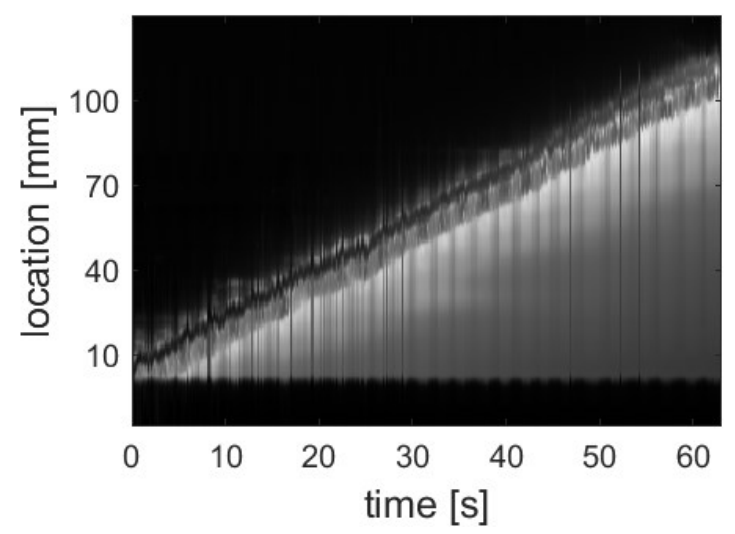

Fig. 9. Welding sequence heatmap extracted from Arwg ROIs for sample with slag inclusions.

For the sample with slag, the plot in which correspondence of welding time and location of electrode tip was generated (Fig. 10). In next step the line was fitted to data. For the presentation purposed the real data was decimated (Fig. 11), but it can be seen that the line fits the data well. It is also confirmed by the $\mathrm{R}^{2}=0.99474$. It can be assumed that the welding speed was constant. 


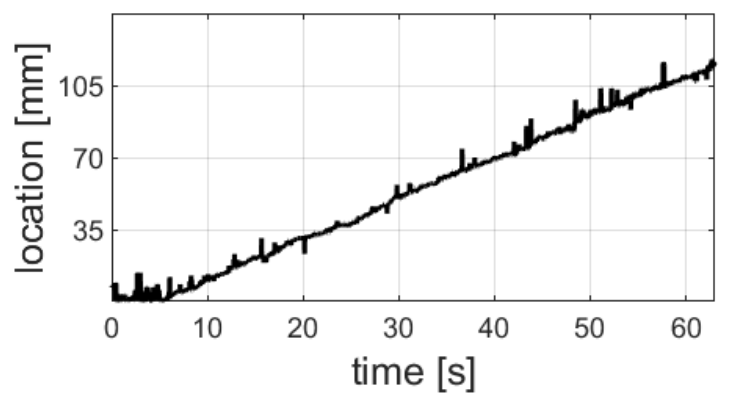

Fig. 10. Welding speed plot for sample with slag inclusions.

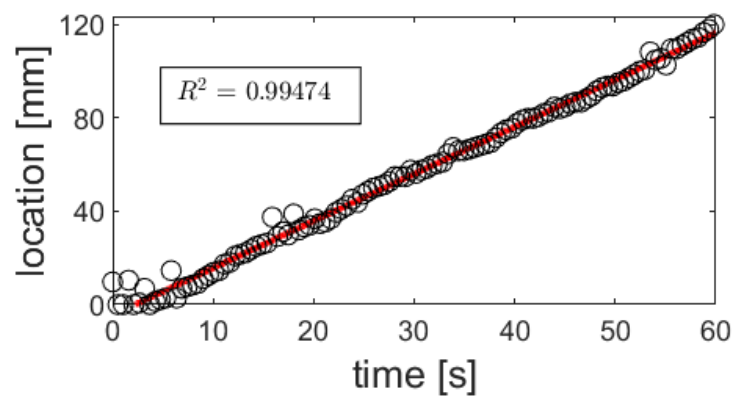

Fig. 11. Welding speed plot for sample with slag inclusions.

The detection signal $\mathrm{tc}_{\mathrm{c}}$ was generated in the way described in sec. 2. For the considered specimen both inclusions were detected and localized properly (Fig. 12). No other detection was made (no false positive) while for all undesired locations the signal was outside (above or below) the detection range. There were also no false negative detections made, so there were no detections in places where the seam was correct.

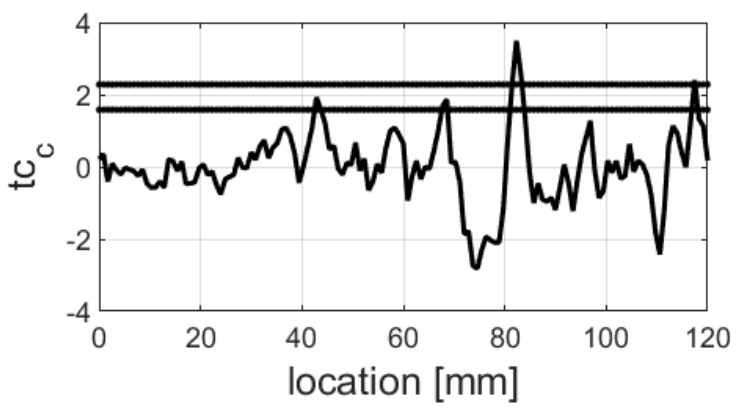

Fig. 12. Slag inclusion detection signal tc $\mathrm{c}_{\mathrm{c}}$ for sample with slag inclusions.

\subsection{Sample without slag}

The weld face and X-ray image of the sample without slag is in Fig. 13. It can be seen that there are no slag inclusions in the layer between seems. On the 9 th $\mathrm{cm}$ there is a large spatter on the bottom side of joint. It is clearly visible on both - photography and the X-ray (the area that is darker than the joint). Other noteworthy spatters are in 2 and $4 \mathrm{~cm}$ of joint length, but they are outside the seam analysis area. 


\section{a)}

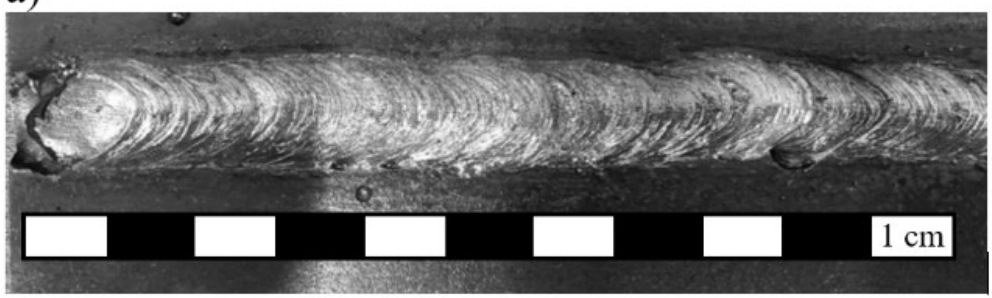

b)

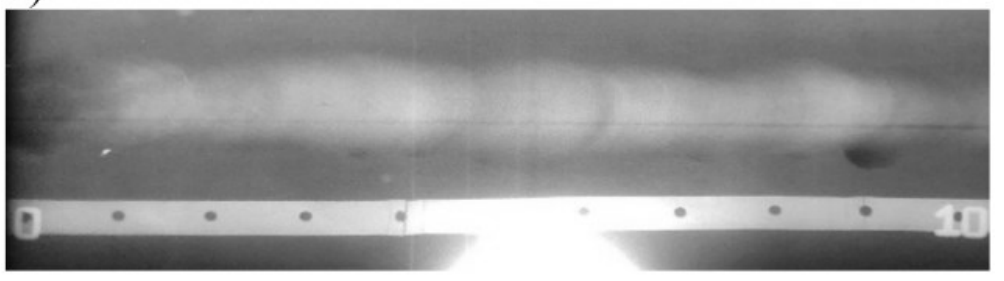

Fig. 13. Welded sample without slag inclusions: a) face view, b) radiogram of joint.

Applying the thermogram sequence procedure, the data recorded during production of the second seam on the specimen, a heat map gathering profiles from $\mathrm{A}_{\mathrm{rwg}}$ area was obtained (Fig. 14). After subjective evaluation it was found that the electrode tip path is close to a straight line. It was further confirmed by $\mathrm{R}^{2}>0.99$.

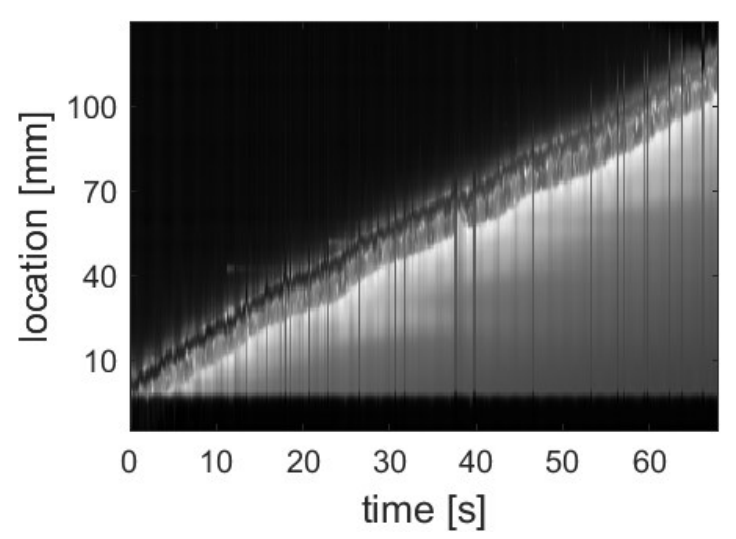

Fig. 14. Welded sample without slag inclusions: a) face view, b) radiogram of joint.

The detection signal $\mathrm{tc}_{\mathrm{c}}$ for the sample without slag inclusion is in Fig. 15. In this case there is one false detection made. In other locations the signal is beyond the detection threshold. It can be also noticed, tat in the first part of the seam process was performed in more sable manner than in the second half. Looking on the weld face it can be found that the weld face width is varying and the surface is rougher in the second part of joint $(6-11 \mathrm{~cm})$. 


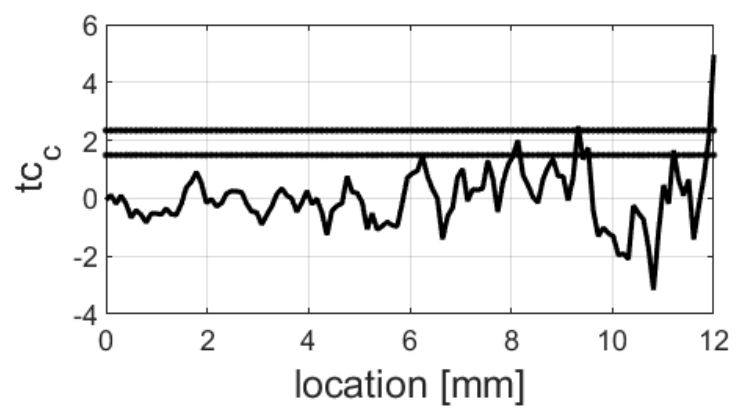

Fig. 15. Slag inclusion detection signal tc $\mathrm{c}$ for sample without slag inclusions.

\section{Conclusion}

In the paper first results of the research concerning the possibility of application of fast detection and localization of slag inclusion that is based on an image processing in signal domain. It is a key assumption to provide a fast and robust method, that is not computationally demanding like methods, where images are transformed into other domains, e.g. frequency domain. Proposed method was elaborated for manual MMA welding, thus verification of welding speed is needed to keep the straightforward correspondence between welding time and electrode tip location. Using proposed regression based method the welding speed variation can be checked. Processing of profiles extracted from the IR image ROIs covering part of produced seam resulting in $t_{c}$ signal that can be used to detect an localize inclusion. The main advantage of this approach is that it can be applied on line, taking into consideration only small parts of the joint, for which the speed check has to be performed and then the detection signal elaborated. Looking on the results obtained for the relatively small set of only eight samples the method has the potential to be a useful tool for slag inclusion detection. There were no false negative detections made, and a small number false positive ones. To better asses the method performance and generalize results larger set of test specimens should be made and investigated. Moreover the minimal size of slag that can be detected with a fixed IR camera setup should be also specified. After research made some additional remarks can be made. False positive recognitions made on the sample without slag relate to the spatter generation during the MMA process. When the sequence of thermograms acquired during the welding procedure was analysed in detail, it was found that directly after the spatter was released from the welding arc area and it appeared in the welding pool it was a similar phenomenon to the pass over the unmelted slag. As the slag remains in the joint the spatter moves over the joint to take final placement outside the seam. This is a fine remark, that to increase the overall method performance and to avoid such type of false positive detection method that will distinguish spatter and inclusions should be developed.

\section{References}

1. P. Moore, G. Booth, Welding problems and defects, The Welding Engineers Guide to Fracture and Fatigue, (2015)

2. K. Weman, Manual metal arc (MMA) welding with coated electrodes, In: Weman K. ed., Woodhead Publishing Series in Welding and Other Joining Technologies, Welding Processes Handbook (Second Edition), Woodhead Publishing, (2012)

3. J. Tomków, D. Fydrych, G. Rogalski, Materials, 12(20), (2019) 
4. J. Tomków, G. Rogalski, D. Fydrych, J. Labanowski, Materials, 16(6), (2019)

5. T. Węgrzyn, R. Wieszala, Arch. Metall. Mater. 57(1), (2012)

6. S. Alfaro, J. Vargas, G. De Carvalho, G. De Souza, J. Mater. Process. Technol. 233, (2015)

7. D. Du, G. Cai, Y. Tian, R. Hou, L. Wang, Robotic Welding, Intelligence and Automation. Lecture Notes in Control and Information Sciences, 362, Springer, Berlin, Heidelberg, (2007)

8. M. Fidali, W. Jamrozik, Infrared Physics \& Technology, 61, (2013)

9. Y. Huang, K. Wang, Q. Zhou et al. Int. J. Adv. Manuf. Technol. 92, (2017)

10. W. Jamrozik, J. Górka, M. Kiel-Jamrozik, Diagnostyka 21, (2020)

11. L. Zhang, A. Basantes-Defaz, D. Ozevin et al. Int. J. Adv. Manuf. Technol. 101, (2019)

12. Y. Zhang, N. Zhang, D. You, X. Gao, S. Katayama, J. Manuf. Processes, 41, (2019)

13. Z. Zhang, H. Chen, Y. Xu, J. Zhong, Mech. Syst. Signal Process, 60, (2015)

14. M. Żuk, J. Górka, R. Dojka, A. Czupryński, Materials Science and Engineering, 227, (2017)

15. W. Jamrozik, Int. J. Mater. Prod. Technol. 51(3), (2015) 